\title{
Nanopesticides: Synthesis and Application of Nanosphare Structures
}

\author{
Vahid Derakhsh AHMADI ${ }^{1}{ }^{*}$, Babak Heidary ALIZADEH $^{2}$ \\ ${ }^{1}$ Department of Plant Protection, Agriculture College, Islamic Azad University, Arak Branch, Iran \\ ${ }^{2}$ Department of Pesticides, Iranian Research Institute of Plant Protection, Iran \\ *Corresponding Author: versasanaatsabz@yahoo.com
}

\begin{abstract}
Nanostructures are of great interest in many current emerging of technology. This review is devoted to the progress made in the last decade in synthesis and applications of nanostructures. We present an overview of synthetic strategies for hollow structures. These strategies are broadly categorized into four themes, which include sell-established approaches, such as conventional hard-templating and soft-templating methods, as well as newly emerging methods based on sacrificial templating and template-free synthesis. The Review therefore focuses on the fundamentals of each process, pointing out advantages and disadvantages where appropriate. Strategies for generating more complex hollow structures, such as nonspherical hollow structures, are also discussed and reviewed.
\end{abstract}

Keywords: template, synthesis, nanosphare

\section{Introduction}

Liquid Suspensions of particles are the basis for an astounding array arrange of materials processes and important in technology. This technology have various applications in different industry including, ceramics, cosmetics, detergents, inks and paints, milk and associated foods and have played a pivotal role in our life (LizMarzan, et al. 1996 and Matijevic, et al. 1993). One of the important advantageous nanostructure composite materials is the impact of particles by combing their functionality with the facile processes of synthetic polymers. In many cases, the nanoparticles play important roles in determining function in a growing number of applications, including catalysis, cosmetics, drug and gene delivery, hydrogen production and storage, photonics, and rechargeable batteries (Caruso, et al 1998). Furtheremore, From 2001, there has been a large increase in research activity on synthesis of hollow nanostructures based on templates (Kresge, 1992, Caruso, 2000, Figure -1).

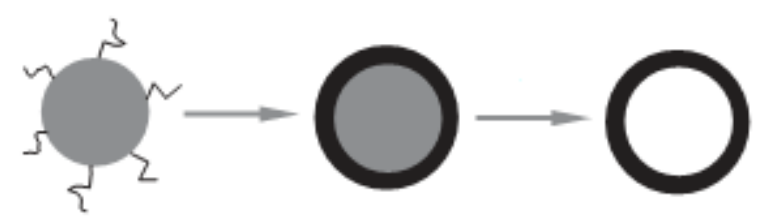

Figure 1: Diagram of synthesis of nanosphare through hard template 
These progresses have in turn motivated applications and fundamental research on nanomaterials in a wide range of fields, such as biomedical engineering, chemical catalysis, energy storage, and photonics. In recent decade, production of nanospher with the sizes between $10 \mathrm{~nm}$ to $10 \mathrm{~mm}$ was attractive. Reviews of specialized nanomaterials of the literature, for example, synthesis of core/shell structures (Caruso, 2001) or synthesis of hollow particles based on specific approaches, such as the Kirkendall effect, (Fan, 2007) Ostwald ripening,(Zeng, 2007, 2006) and layer-by-layer (LBL) assembly, We divide these approaches into four categories.

\subsection{Conventional Hard Templates}

Synthesis of hollow structures by templating through hard particles is conceptually simple. In general, it involves the four major steps: (1) preparation of hard templates; (2) functionalization of template surface to accomplish favorable surface properties; (3) coating the templates with designed materials by various processes, possibly with post-treatment to form compact shells; and (4) selective removal of the templates to obtain hollow structures (Figure-1). The most commonly used hard templates include nearly monodisperse silica particles and polymer latex colloids (Xu, 2004 and Guan, 2007). These templates are favorable for several reasons including their narrow size distribution, and simplicity of their synthesis, availability in a wide range of sizes from commercial sources and availability in relatively large amounts. Other colloids, such as carbon nanospheres and nanoparticles of metals and metal oxides, have also been used as templates for preparation of hollow structures. It normally involves selective remove of the template in proper solvents or calcinations of the template at high temperatures. Using either path, special care is required to prevent collapse of the wells during template removal. For example, when using organic solvent to dissolve template latex particles, swelling of the polymer can cause burst of the hollow structure, causing the shell to manifest uncontrolled shape change. Step 3 is usually consider as the most difficult step because it requires potent methods for efficiently precipitating shell materials on substrates with sizes in the nanometer range. Incompatibility between the template surface and shell material can be improve by modifying the template with special functional groups or characteristics, for example electrostatic charges, before applying subsequent coating/ precipitation of shell material Step 2 and Step 3 together therefore constitute a coating scheme to form compact shells on any arbitrary template particles. Unsurprisingly, the most exciting research in the field has focused on developing flexible coating schemes suitable for a broad range of materials. This attempt has led to considerable progress during the past decade. While organic polymer hollow structures are also often prepared using different hard templates in the following, we will focus on the shellforming procedure for inorganic or hybrid material

\subsection{Layer-by-Layer Assembly}

LBL assembly refers to consecutive deposition of oppositely charged polymer on substrates settle by electrostatic interactions. Combining this procedure with colloidal templating, Caruso et al, (2000) were the first to develop the LBL technique to prepare hollow inorganic silica and hybrid capsules from electrostatic association of negatively charged silica 
nanoparticles and positively charged polymer. Figure-2 shows the basic rout using polystyrene (PS) latex particles as templates. The formation cycle can be repeated to assemble multilayer with well-defined wall thickness (Zelikin, 2006). The utility of the LBL method is one of its main advantages. It also promotes excellent capsule size control and uniformity of the well. Polymer capsules synthesised from this route enable the encapsulation of several components (e.g., DNA) and are presently of special interest in bioapplications. Other than various polymer capsules, the LBL technique has also been extensively used to synthesized hollow structures of a broad range of inorganic or composite materials (Wang, et al, 2004) .

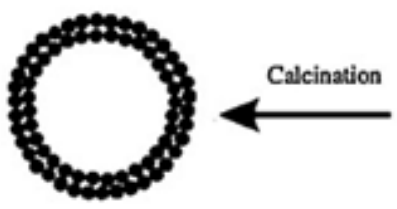

Silica Spheres

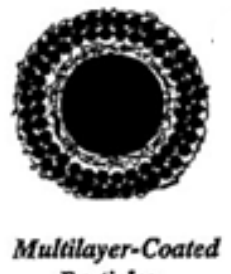

Particles

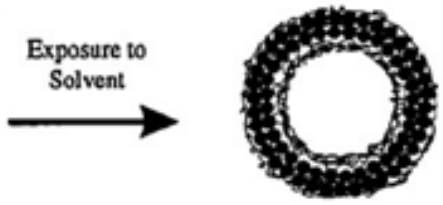

Inorganic-Hybrid Spheres

Figure 2: Hybrid nano silica through layer-layer polystyrene hard template.

Although its flexibility, the LBL method suffers from two key shortcomings. First, the method is difficult to use for preparation of smaller hollow structures with sizes $<200 \mathrm{~nm}$. Second, the LBL formation procedure becomes quite tedious when many layers are required.

\subsection{Direct Chemical Deposition}

Chemical deposition is also a commonly used well-forming. It involves precipitation of the shell materials or precursors on the template particles from various chemical or physical relations with the template. The deposition process is normally followed by a post-treatment (usually calcination) step to obtain compact shells (figure-3)

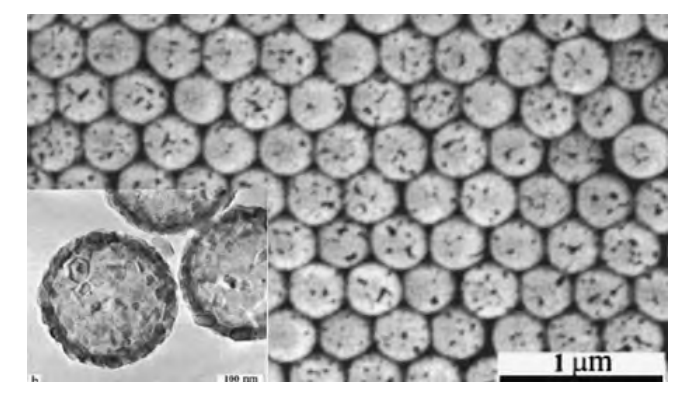

Figure 3: TEM of nanosphare of titania covered by PS

Control of the hydrolysis rate and hetero coagulation is necessary for the success of the sunthesis. Imhof (2001) carried out the coating in ethanol at room temperature by hydrolysis of titanium tetraisopropoxide using cationic polystyrene (PS) spheres as templates stabilized by polyvinylpyrrolidone (PVP). The slight negative charge on the hydrolyzed titania species ensures their rapid deposition on the positively charged PS spheres, although the hydrolysis of titanium alkoxide is rather fast. As-formed titania is amorphous, and post-calcination at high temperatures is usually employed to accompanying crystallize the particles and remove 
the polymer templates. Shrinkage during the crystallization action and, escape of gases produced by pyrolysis of the polymer templates, often lead to gap such as holes on surfaces of the final hollow particles. A particularly worthwhile application of this strategy is found in the synthesis of hollow $\mathrm{SiO}_{2}$ nanostructures. Uniform deposition of $\mathrm{SiO}_{2}$ from hydrolysis of tetraethoxysilane (TEOS) is known to be relatively easy, particularly on inorganic templates (Graf, 2003). As a result, smooth $\mathrm{SiO}_{2}$ coating on particles of great materials has been recorded and for example, mesoporous $\mathrm{Fe}_{3} \mathrm{O}_{4}$ hollow spheres have been prepared by chemical adsorption/precipitation of $\mathrm{Fe}^{+}$on carboxyl-functionalized PS templates at high temperaturw in the presence of ethylene glycol (EG).

\subsection{Chemical Adsorption on Surface Layer}

Nano spheres have also been prepared by many groups applying a direct adsorptioncalcination route. In this method, pretreatment of the template particles is usually required (Kim, 2002). Hyeon et al. have prepared Pd hollow spheres as shown in Figure 4. This strategy is normally for hard-templating fabrication of hollow spheres. However, formation of a complete shell after template removal is quite uncommon because the adsorbed precursor amount is rather limited on the monolayer functionalized surface.

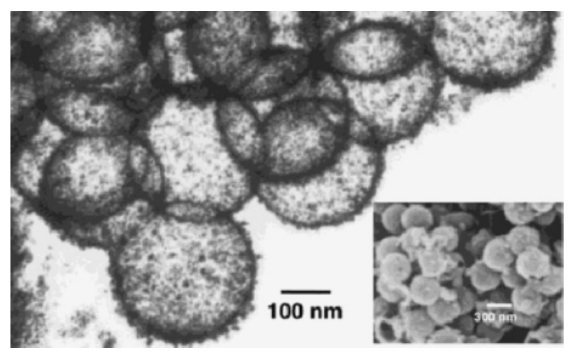

Figure 4: TEM and SEM of palladium nanoshpare

1.5. Nano spheres with mesoporous shells (HSMS)

As shown in Figure -5, Nano spheres with mesoporous shells (HSMS) can be synthesised using spherical templates with solid core and mesoporous shell (SCMS) farme (Stober, 1968). SCMS silica templates are most typically used, which can be regularly prepared by forming a suitable agent (e.g., n-octadecyltrimethoxysilane) during the routine synthesis of silica particles by the Stober's method (Ikeda et al., 2007).

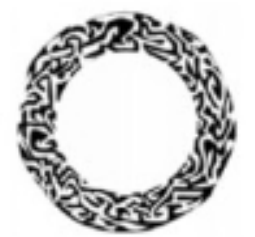

Figure 5: Nanoshare with mesoporous structure 


\subsection{Templating against Colloidal Crystals and the Lost-Wax Approach}

If a sol-gel starting material is penetrate into a physically stable colloidal, generally expect to obtain a 3D porous replica. Electrostatic repulsion between the charged PS particles ensures that they remain separated and are surrounded by the sol-gel material solution (Zhong, 2000). Once treated to moisture in air, the sol-gel material hydrolyzes quickly to form the related metal oxide sols, which discharged on the "sticky" surface of PS particles (Figure-6). As solvent removes, the precipitate forms a uniform coating on the PS template. Keep other conditions unchanged, the well thickness is mainly determined by the concentration of solgel starting materials. The achievement of this method evidently requires good control of the interfacial properties of both the polymer materials and sol-gel compounds, as well as of the hydrolysis rate of the starting materials.

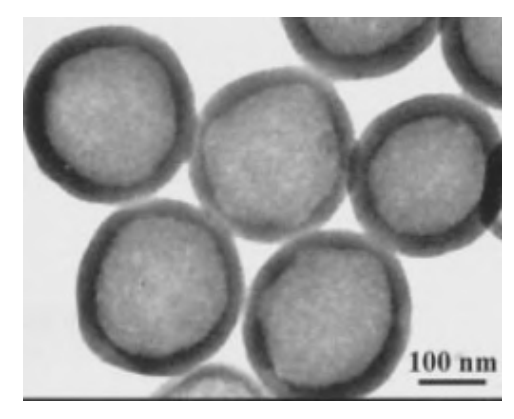

Figure 6: Nanoclloidal of Titania through Polystyrene template

\section{Sacrificial Templates}

These template itself is elaborated as a reactant in the synthetic process of the well material or its intermediate (Smigelskas, 1947). In this concern, sacrificial template synthesis is intrinsically advantageous because in general it needs no additional surface functionalization and well formation is assured by chemical reaction (Figure-7).

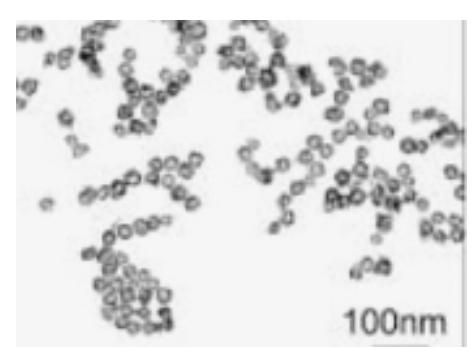

Figure 7: Nanoshare of platin through cobalt sacrificial template

\section{Soft Templates}

Templating against hard (solid) templates is conceivably the most effective, and certainly the most common, method for synthesizing hollow micro. However, hard templates have several inherent disadvantages, which range from the intrinsic difficulty of performing high product yields from the multistep synthetic process to the lack of structural strength of the shells upon template removal (Ni, 2006, Zimmermann, 2007). Also, key applications of hollow structures, such as drug and remedial delivery, require facile access to the nano interior space. 
With the hard templates, refilling the hollow interior with functional species or in situ encapsulation of guest molecules during formation of the walls, though possible, is very challenging. These difficulties have stimulate interest in simpler synthetic routes for producing hollow shells that consent easy encapsulation and release of guest materials. Among these paths, templating against soft templates has attracted the greatest attention and important progress has been made in the past decade. In this section, we will review commonly used soft templates, including emulsion droplets, surfactant and other supramolecular micelles, polymer aggregates/vesicles, and gas bubbles, and explain their application to synthesis of nanostructures.

\subsection{Emulsion Droplets}

When two immiscible liquids are mixed together through mechanical stirring, liquid droplets of one phase can be diffuse in the other continuous phase, forming an emulsion. In general, emulsions are thermodynamically unstable, thus surfactants or amphiphilic polymers, which places at the interface between the droplets and continuous phase, are required to increase kinetic stability. Oil-in-water or water-in-oil emulsions are most frequently used. It should be mentioned that emulsion polymerization, where the droplets act as microreactors, has long been used to synthesize solid and hollow polymer spheres. Sol-gel proceeding for metal alkoxides are usually used in emulsion templating to prepare hollow spheres of metal oxides such as silica and $\mathrm{TiO} 2$. Li et al. (2003) prepared hollow spheres of aluminosilicate with mesoporous shells by combining emulsion droplet templating and surfactant templating. The $\mathrm{O} / \mathrm{W}$ emulsion is produced by adding TEOS into aqueous $\mathrm{Al} 2\left(\mathrm{SO}_{4}\right)_{3}$ solution (Figure-8). The surfactant $(\mathrm{CTAB})$ molecules are responsible for stabilization of the droplets and templating the mesoporous wall formation.

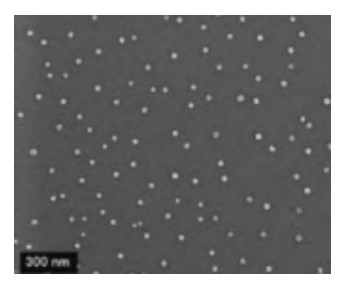

Figure 8: SEM of nanosphare $\gamma-\mathrm{AlO}(\mathrm{OH})$

Usually, it is very confront to obtain uniform emulsion droplets with a small size $<100 \mathrm{~nm}$ because of droplet coalescence and Ostwald ripening (Imhof, 1997). Most recently, Feldmann and co-worker synthesized boehmite hollow nanospheres through hydrolysis of $\mathrm{Al}(\mathrm{sec}-$ $\left.\mathrm{OC}_{4} \mathrm{H}_{9}\right) 3$ via a W/O (n-dodecane) emulsion templating procedure. The emulsion droplets are stabilized by CTAB. As a result, the as-prepared boehmite nanospheres with size of about 30 $\mathrm{nm}$ are quite homogeneous.

\subsection{Supramolecular Micelles/Vesicles}

Amphiphilic molecules such as surfactants can be made to collect into micelles or vesicles with different structures when their concentration in solution exceeds some critical values (Fendler, 1980). For example, at concentrations above ca. $8.2 \mathrm{mM}$ at $25^{\circ} \mathrm{C}$, the so-called 
critical micelle concentration (CMC) in an aqueous solution, sodium dedecyl sulfate (SDS) will spontaneously form spherical micelles, each containing about 50 molecules arranged in a regular radial pattern. In general, the system and stability of micelles or vesicles are affected by many factors such as the solvent polarity, $\mathrm{pH}$ value, and ionic strength of the solution. This sensitivity of micelle/ vesicle structure to easily controlled synthesis conditions can in principle provide elegant control over template shape and structure. First, the sol-gel chemistries for hydrolysis and condensation of silicon alkoxides in aqueous solution can be well controlled. Second, it assets from the excessive development in the field of surfactant directed synthesis of arranged mesoporous silica, for example, MCM-41. Pinnavaia (Kim, 1998) and co-workers synthesised stable silica vesicles with the shells constructed of lamellar sheets (see Fig. 9). The method is based on supramolecular assembly by hydrogen bonding between neutral surfactants of the type $\mathrm{RNH}\left(\mathrm{CH}_{2}\right)_{2} \mathrm{NH}_{2}$ and silica precursors derived from TEOS. Hubert et al. (2000) reported the first successful deposition of silica on unilamellar surfactant of dioctadecyldimethylammonium bromide by mild hydrolysis and condensation of silicon alkoxides.

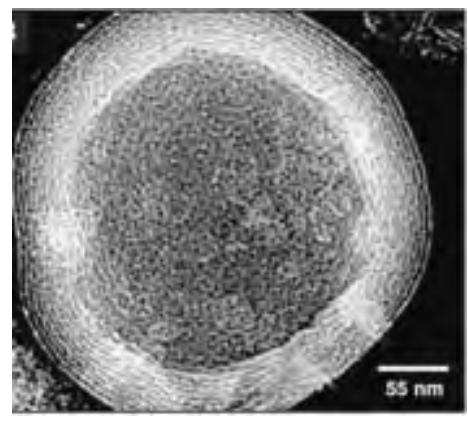

Figure 9: TEM of nanovesicules with layer-layer shells

\subsection{Polymer Aggregates/Vesicles}

As mentioned in the preceding section, the structure and stability of surfactant micelles are sensitive to many parameters, such as $\mathrm{pH}$, temperature, concentration, solvent, additives, and ionic strength. As a result, common use of surfactant phases to template hollow particles remains assert. Outstandingly, in some cases these difficulties can be resolved using more complex systems, such as aggregates of surfactant-polymer mixtures or even charged polymers (Scaiano and Abuin 1984). Qi and co-workers have used the complex poly(ethylene oxide)-block-poly(methacrylic acid)-sodium dodecylsulfate micelles (PEO-bPMAA-SDS) as soft template to synthesized $\mathrm{CaCO}_{3}$ and $\mathrm{Ag}$ nanospheres (Figure-10). 


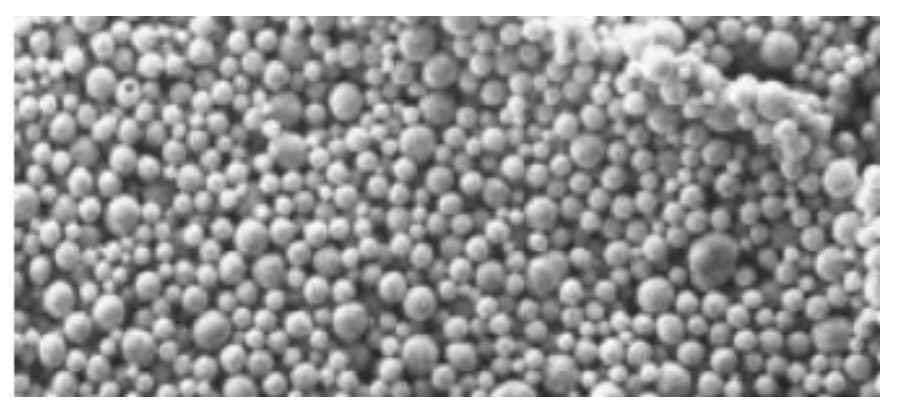

Figure 10: SEM of complex nanosilver with PEO-b-PMAA-SDS

It is common that PEO can interact with anionic SDS to form PEO- SDS complex micelles with EO groups intermingling in the head group region of the SDS micelles. Ding et al. (2004) prepared $\mathrm{Fe}_{3} \mathrm{O}_{4}$-polymer hybrid hollow nanospheres through polymerization and cross-linking of acrylic acid (AA) in the wall of micelles self-assembled from cationic chitosan and anionic AA preloaded with poly(vinyl alcohol) stabilized $\mathrm{Fe}_{3} \mathrm{O}_{4}$ nanoparticles (Hu, 2004). After removing the polymer at high temperature under argon, relatively uniform $\mathrm{Fe}_{3} \mathrm{O}_{4}$ hollow nanospheres can be obtained.

\subsection{Gas Bubbles}

Gas bubbles diffused in a liquid host can be applied to produce stable emulsions and foams, which have recently appear as promising soft templates for synthesis of an increasing number of hollow particles (Peng, et al, 2003). In general, the procedure can be approach in three steps as shown in, formation of fine nanoparticles and gas bubbles, coupling of fine nanoparticles on the liquid interface, and further aggregation of nanoparticles forming compact shells around the gas bubble. Han et al. prepared $\mathrm{CaCO}_{3}$ hollow spheres by blowing a mixed gas $\left(\mathrm{CO}_{2}\right.$ and $\left.\mathrm{N}_{2}\right)$ into a solution of $\mathrm{CaCl}_{2}$ and ammonia (Figure-11).

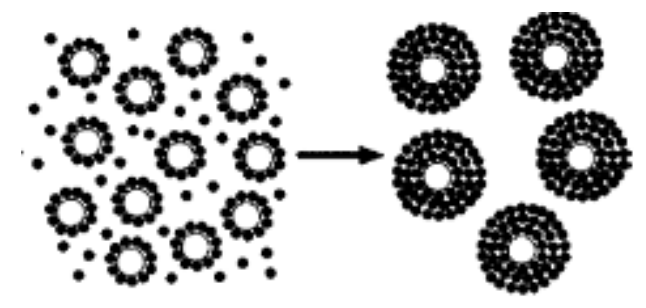

Figure 11: Nanosphare through gas template

\subsection{Template-Free Methods}

Disadvantages related to high cost and exhausting synthetic procedures have deter scale-up of many of these methods for large scale applications. The synthesis is performed in an ethanolwater mixed solvent using potassium stannate $\left(\mathrm{K}_{2} \mathrm{SnO}_{3}{ }_{3} \mathrm{H}_{2} \mathrm{O}\right)$ as the precursor $(\mathrm{Li}, 2006)$. 


\section{Conclusion}

Recently, many progresses have been made in synthesis of nanostructure in many area of technology. The successes in synthesis of hollow structures through temple have provided opportunities to tune their mechanical, optical, electrical, chemical, and other properties. These advances have in turn catalyzed exploration in a growing list of applications.

\section{References}

[1] Abuin, B., Scaiano, J. C. 1984 J. Am. Chem. Soc., 106, 6274. Adv. Mater.,19, 2370.

[2] Caruso, F. 2000 Chem. Eur. J.,6, 413.

[3] Caruso, F., Adv. Mater. 2001, 13, 11

[4] Caruso, F., Caruso, R. A., Mohwald, H. 1998 Science, 282, 1111.

[5] Ding,Y., Hu, Y., Jiang, X. Q., Zhang, L. Y. Yang, C. Z., Angew. Chem. Int. Ed. 2004, 43, 6369

[6] Fan,H. J., Gosele, U., Zacharias, M., Small 2007, 3, 1660

[7] Fendler, J. H. Acc. Chem. Res. 1980,13, 7.

[8] Graf, C., Vossen, D. L. J., Imhof, A., Blaaderen, A. van, Langmuir 2003, 19, 6693.

[9] Guan, G. J., Zhang, Z. P., Wang, Z. Y., Liu, B. H., Gao, D. M., Xie, C. G. 2007

[10] Han, Y. S., Hadiko, G., Fuji, M., Takahashi, M., Chem. Lett. 2005, 152.

[11] Hu, Y. Jiang, X. Q., Ding, Y., Chen, Q., Yang, C. Z., Adv. Mater. 2004, 16, 933.

[12] Hubert, D. H. W., Jung, M., Frederik, P. M., Bomans, P. H. H., Meuldijk, J., German, A. L. $2000 A d v$. Mater.,12, 1286.

[13] Ikeda, S., Tachi, K., Ng, Y. H., Ikoma, Y., Sakata, T., Mori, H., Harada, T., Matsumura, M. 2007 Chem. Mater.,19, 4335.

[14] Imhof, A., Langmuir 2001, 17, 3579.

[15] Imhof, D. J. Pine, J. Colloid Interface Sci. 1997, 192, 368

[16] Kim, S. W., Kim, M., Lee, W. Y., Hyeon, T. 2002 J. Am. Chem. Soc., 124, 7642.

[17] Kim, S. W., Zhang, S. Z., Pinnavaia, T. J. 1998 Science, 282, 1302

[18] Kresge, C. T., Leonowicz, M. E., Roth, W. J., Vartuli, J. C., Beck, J. S. 1992 Nature, $359,710$.

[19] Li, B. X., Rong, G. X., Xie, Y., Huang, L. F., Feng, C. Q. 2006 Inorg. Chem.,45, 6404.

[20] Li, Y. S., Shi, J. L., Hua, Z. L., Chen, H. R., Ruan, M. L., Yan, D. S. 2003 Nano Lett.,3, 609.

[21] LizMarzan, L. M., Giersig, M., Mulvaney, P. 1996 Langmuir,12, 4329.

[22] Matijevic, E. 1993 Chem. Mater.,5, 412.

[23] Ni, Y. H., Tao, A., Hu, G. Z., Cao, X. F., Wei, X. W., Yang, Z. S. 2006 Nanotechnology, 17, 5013. 
[24] Peng, Q., Dong, Y. J., Li, Y. D. 2003 Angew. Chem. Int. Ed.,42, 3027.

[25] Smigelskas, A. D., Kirkendall, E. O., 1947 Trans. Am. Inst. Min. Metall. Pet. Eng., 171, 130.

[26] Stober, W., Fink, A., Bohn, E., J. 1968 Colloid Interface Sci.,26, 62.

[27] Wang, L. Z., Ebina, Y., Takada, K., Sasaki, T. 2004 Chem. Commun., 1074.

[28] Xu, X. L., Asher, S. A. 2004 J Am. Chem. Soc., 126, 7940.

[29] Z. Y. Zhong, Y. D. Yin, B. Gates, Y. N. Xia, 2000 Adv. Mater., 12, 206.

[30] Zelikin, N., Li, Q., Caruso, F. 2006 Angew. Chem. Int. Ed.,45, 7743.

[31] Zeng, H. C., Curr. Nanosci. 2007, 3, 177.

[32] Zeng, H. C., J. Mater. Chem. 2006, 16, 649

[33] Zimmermann, C., Feldmann, C., Wanner, M., Gerthsen, D. 2007 Small, 3, 1347. 